\title{
Clear Cell Variant of Papillary Thyroid Carcinoma: A Case Report
}

\author{
Fahad Naser Algahtani ${ }^{\mathrm{a}}$, Khalid Saud Alshaalan ${ }^{\mathrm{a}, \mathrm{d}}$, \\ Majed Pharaon ${ }^{\mathrm{b}}$, Mohammed Elkrim ${ }^{\mathrm{c}}$
}

\begin{abstract}
Clear cell variant of papillary thyroid carcinoma is considered a very rare morphologic variant. The incidence of clear cell papillary thyroid cancer is estimated to be around $0.5 \%$. Due to its rarity, currently there is a lack of knowledge about this variant; especially the clinical importance, management approach and prognosis. This is a case of a 64-year-old female; she was diagnosed with clear cell variant of papillary thyroid carcinoma. This report describes the clear cell variant microscopically and elaborates on the key features of this variant. Moreover, this report explores the clinical conditions of this case, discusses the management plan, and the prognosis of clear cell variant of papillary thyroid carcinoma.
\end{abstract}

Keywords: Clear cell variant thyroid carcinoma; Clear cell papillary thyroid carcinoma; Papillary thyroid carcinoma, thyroid carcinoma

\section{Introduction}

Papillary thyroid carcinoma (PTC) is considered the most common type of thyroid malignancies, which accounts for about $70 \%$ of thyroid malignancies. Exposure to radiation and genetic susceptibility are commonest risk factors related to it. PTC has many variants. Some of these variants are common like conventional, follicular and tall cell variants; however, the other variants are rare, such as columnar cell, diffuse sclerosing and clear cell variants [1]. Due to the variety of the histopathologic variants of PTC and the lack of well-defined classification, determination of the variant can be challenging.

\footnotetext{
Manuscript submitted March 6, 2019, accepted April 8, 2019

${ }^{a}$ College of Medicine, King Saud bin Abdulaziz University for Health Sciences, Riyadh, Saudi Arabia

${ }^{b}$ Department of Pathology, King Abdulaziz Medical City, National Guard Health Affairs, Riyadh, Saudi Arabia

'Division of Otolaryngology-Head and Neck Surgery, Department of Surgery, King Abdulaziz Medical City, National Guard Health Affairs, Riyadh, Saud Arabia

${ }^{\mathrm{d} C o r r e s p o n d i n g ~ A u t h o r: ~ K h a l i d ~ A l s h a a l a n, ~ C o l l e g e ~ o f ~ M e d i c i n e, ~ K i n g ~ S a u d ~}$ bin Abdulaziz University for Health Sciences, Riyadh, Saudi Arabia. Email: Alshaalankh@ngha.med.sa
}

Moreover, some guidelines classify these variants according to the size like papillary microcarcinoma and papillary macrocarcinoma; and according to their architecture like follicular, macrofollicular, cribriform-morular, solid and micropapillary; and others are classified according to their cellular characteristics like tall, columnar, oncocytic, clear and hobnail cell. Adding to that, some carcinomas consist of more than one PTC variant, which complicates the classification furthermore. Although most variants do not have clinical implications, some histological variants are important to be identified due to its prognostic values, and it could exemplify the associated clinical disorders [2].

\section{Case Report}

A 64 years old female, known case of diabetes mellitus and hypertension presented to emergency department vitally stable with a history of left neck mass for 1 week, with changes in her voice and difficulty in swallowing. On examination, the neck mass was tender, mobile, regular and about $4 \times 3 \mathrm{~cm}$ in size. A computed tomography (CT) scan was ordered to rule out retropharyngeal abscess; then the case referred to otolaryngology for further management. The CT scan showed a left thyroid lobe lesion with large left carotid space lymph node masses, which was worrisome for malignancy (Fig 1.). An ultrasoundguided fine needle aspiration (FNA) was obtained from the left thyroid nodule that showed conventional type PTC, arising in a background of Hashimoto's thyroiditis. Moreover, the

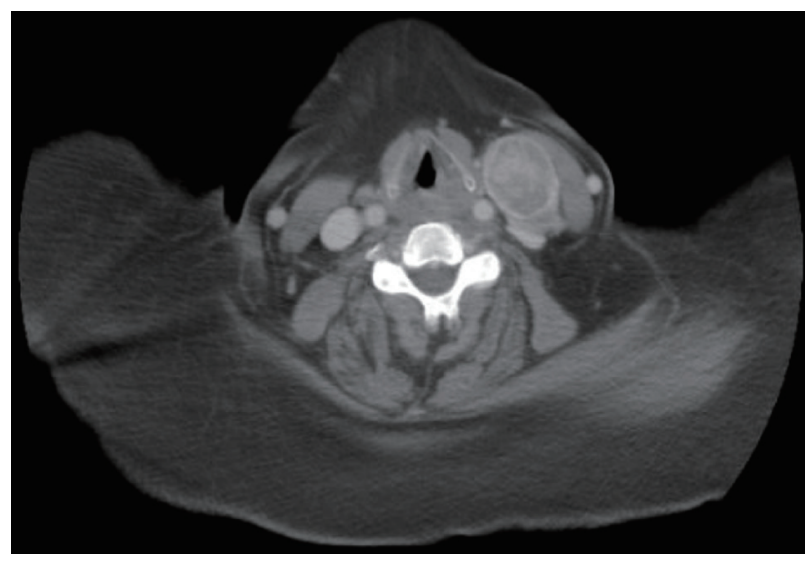

Figure 1. Neck computed tomography scan. 


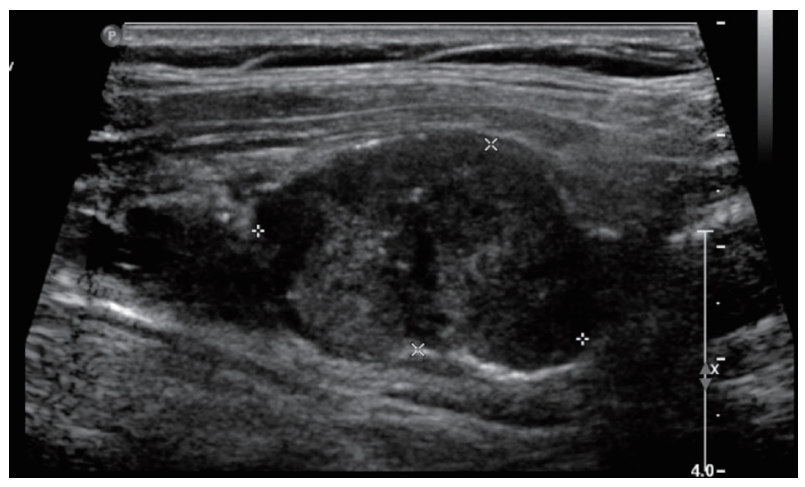

Figure 2. Neck ultrasonography for the neck mass.

FNA sample from the left lymph node was consistent with metastatic cystic PTC. Another CT was obtained after 5 days to evaluate the metastasis to adjacent structures, which revealed a heterogeneous enlarged left thyroid lobe with calcified nodules and multiple enlarged right supraclavicular and small superior mediastinal calcified lymph nodes without other intrathoracic metastasis. Finally, neck ultrasound was performed and demonstrated the measurement as the following, the thyroid isthmus was $0.6 \mathrm{~cm}$, right thyroid lobe was $4.6 \times 2.2 \times 1.5 \mathrm{~cm}$, and the left thyroid lobe measures $4.8 \times$ $2.6 \times 2.2 \mathrm{~cm}$. There were several, at least three, right thyroid lobe nodules of variable sizes, the largest one was near the isthmus anteriorly, and measuring $0.6 \times 0.4 \mathrm{~cm}$. There was a $2.5 \times 1.6 \mathrm{~cm}$, hypoechoic lesion with central calcification and lobulated margins seen in the left thyroid lobe with questionable extension beyond the thyroid gland. Also, there were two large well-defined complex masses with internal vascularity seen in the left neck, adjacent to the left thyroid lobe and left common carotid artery, measuring $3.8 \times 2.1 \mathrm{~cm}$ and $3 \times 1.9$ $\mathrm{cm}$ respectively (Fig 2.).

After that, the patient went for laryngoscopy and was found to have a complete immobile left vocal cord. At that time, the patient was diagnosed to have stage T4aN1bMX PTC, with left vocal cord palsy. The patient consented for total thyroidectomy, central neck dissection, and lateral left neck dissection from level 2 to 5 . The patient tolerated the procedure well and was in good and stable condition. The surgical pathology specimen that was collected during the surgery showed PTC, clear cell variant. The patient was discharged on levothyroxine, calcium carbonate, and painkillers and was followed up in the outpatient clinic.

\section{Discussion}

Clear cell variant of PTC is considered a rare morphologic variant. The information about the prevalence and incidence of this variant is very limited due to the rarity and the lack of studies. The study of Segal et al which was conducted in the
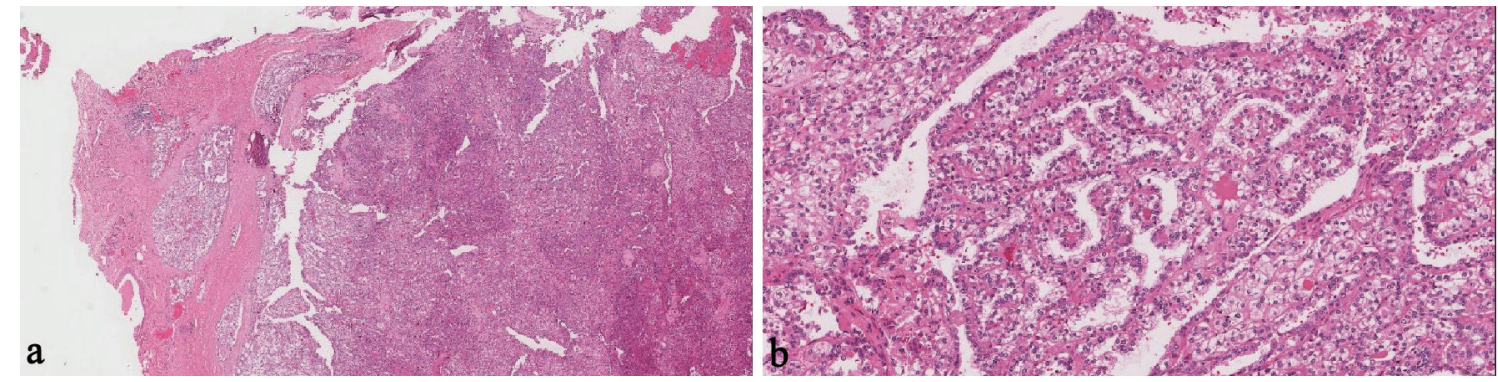

Figure 3. (a) Solid sheets of tumor cells (original magnification: $\times 40$, H\&E stain). (b) Focal papillary architecture (original magnification: $\times 200$, H\&E stain).

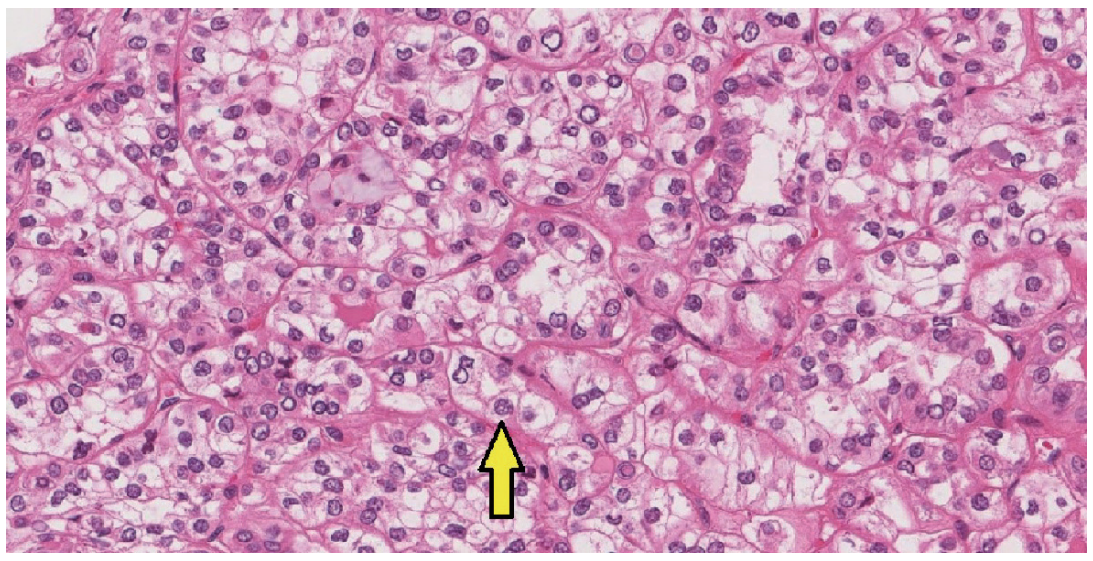

Figure 4. Nuclear features of papillary thyroid carcinoma (original magnification: $\times 400, \mathrm{H} \& \mathrm{E}$ stain, arrow: intranuclear pseudoinclusions). 
USA reported only three cases of clear cell variant which accounts for $0.52 \%$ only [3]. Another study from Japan reported a close percentage $0.18 \%$ [4]. Clear cell carcinoma can affect many organs like vagina, lung, liver, bladder, and thyroid. It has a clear appearance which is due to the abundant glycogen in the cytoplasm in addition to mucin and lipids [5]. According to a study which was conducted in USA [6], there are four possible causes for clear cell carcinoma to occur in thyroid gland: primary follicular derived tumor, clear cell medullary carcinoma of the thyroid, parathyroid tumors, and metastatic clear cell renal cell carcinoma. It is important to differentiate between these different causes. For example, parathyroid tumors could be ruled out by measuring the parathyroid hormone (PTH), and for the clear cell medullary carcinoma, we can measure the calcitonin level. Due to the similarity in the morphological appearance of the primary follicular derived tumor and metastatic clear cell renal cell carcinoma, it is very difficult to differentiate between them, though it is very important to consider the best management plan. Most of the studies agree that staining for thyroglobulin is the most useful tool to differentiate between the two of them, but it has some pitfalls [6].

In our case, the microscopic description of the histologic sections demonstrates multiple foci of papillary thyroid carcinoma scattered within the left lobe and isthmus. Few sections show bands of fibrosis representing previous FNA associated changes. The tumor cells are arranged predominantly in solid sheets with focal papillary configuration. Approximately $80 \%$ of the tumor cells have a striking clear-appearing cytoplasm (Fig. 3a, b). The classic nuclear features of PTC are demonstrated by enlarged overlapped nuclei, chromatin clearing, irregular contours, grooves, and intranuclear cytoplasmic pseudoinclusions (Fig. 4). Currently, the treatment and prognosis of PTC clear cell variant are considered similar to conventional/classic papillary thyroid carcinoma, thus there are no such studies to support this idea. In conclusion, more studies on large population are required to determine the best management and prognosis of the PTC, clear cell variant.

\section{Acknowledgments}

None to declare.

\section{Financial Disclosure}

This study has not received any funding.

\section{Conflict of Interest}

All authors declare that there is no conflict of interest.

\section{Informed Consent}

A written informed consent was obtained from the patient to publish this report.

\section{Author Contributions}

All authors contributed equally in interpretation, writing, reporting and reviewing this case report.

\section{References}

1. Lloyd RV, Buehler D, Khanafshar E. Papillary thyroid carcinoma variants. Head Neck Pathol. 2011;5(1):51-56.

2. Sak SD. Variants of papillary thyroid carcinoma: multiple faces of a familiar tumor. Turk Patoloji Derg. 2015;31(Suppl 1):34-47.

3. Segal K, Har-El G, Avidor I, Shvero J, Lubin E, Sidi J. Clear cell carcinoma of the thyroid gland. Head Neck Surg. 1986;8(4):313-319.

4. Ito Y, Hirokawa M, Uruno T, Kihara M, Higashiyama T, Takamura Y, Miya A, et al. Prevalence and biological behaviour of variants of papillary thyroid carcinoma: experience at a single institute. Pathology. 2008;40(6):617-622.

5. Schroder S, Bocker W. Clear-cell carcinomas of thyroid gland: a clinicopathological study of 13 cases. Histopathology. 1986;10(1):75-89.

6. Shimizu K, Nagahama M, Kitamura Y, Chin K, Kitagawa W, Shibuya T, Mimura T, et al. Clinicopathological study of clear-cell tumors of the thyroid: an evaluation of 22 cases. Surg Today. 1995;25(12):1015-1022. 